\title{
VisualBlock-FIR for Fault Detection and Identification: Application to the DAMADICS Benchmark Problem
}

\author{
Antoni Escobet ${ }^{1}$, Àngela Nebot ${ }^{2}$, and François E. Cellier ${ }^{3}$ \\ ${ }^{1}$ Dept. ESAII, Universitat Politècnica de Catalunya, Manresa, Spain \\ toni @epsem.upc. edu \\ ${ }^{2}$ Dept. LSI, Universitat Politècnica de Catalunya, Barcelona, Spain \\ angela@lsi.upc.edu \\ ${ }^{3}$ Institute of Computational Science, ETH Zurich, CH-8092 Zurich, Switzerland \\ FCellier@Inf.ETHZ.CH
}

\begin{abstract}
This paper describes a fault diagnosis system (FDS) for non-linear plants based on fuzzy logic. The proposed scheme, named VisualBlock-FIR, runs under the Simulink framework and enables early fault detection and identification. During fault detection, the FDS should recognize that the plant behavior is abnormal, and therefore, that the plant is not working properly. During fault identification, the FDS should conclude which type of failure has occurred. The enveloping and acceptability measures introduced in VisualBlockFIR enhance the robustness of the overall process. The final part of this research shows how the proposed approach is used for tackling faults of the DAMADICS benchmark.
\end{abstract}

\section{Introduction}

There has been an intensive research activity in the Fault Diagnosis System (FDS) area that includes quantitative as well as qualitative approaches. Quantitative approaches are primarily based on statistical techniques, first order logic, control theory, mathematical modeling, and computer simulation $[1,2,3]$. The main drawback of quantitative techniques is that they operate on a quantitative and precisely formulated plant model that is not always available. Also, human plant operators usually rely on heuristic knowledge that is easy to be captured by means of qualitative methodologies. There is a large amount of research done in the area of qualitative FDS, specially using expert systems, neural networks, and genetic programming [4, 5, 6, 7]. However in recent years, the demand has arisen to develop FDS that are more robust to uncertainty. In this context, fuzzy logic and hybrid fuzzy approaches appear to offer a good alternative to other qualitative FDS methodologies $[8,9,10]$.

In this paper, a VisualBlock-FIR FDS based on fuzzy inductive reasoning (FIR) methodology is presented and applied to a pneumatic servomotor actuated control valve, which is the benchmark problem of the European research training network called DAMADICS. DAMADICS stands for Development and Application of Methods for Actuator Diagnosis in Industrial Control Systems.

A. Gelbukh and A.F. Kuri Morales (Eds.): MICAI 2007, LNAI 4827, pp. 1173-1183, 2007.

(C) Springer-Verlag Berlin Heidelberg 2007 
In the next section, the fault detection and identification processes of VisualBlockFIR are introduced. Section 3 presents the case study and the results obtained. Finally, the conclusions of the research are presented in section 4 .

\section{Fault Detection and Identification Methodology}

As mentioned before, a FMS needs to detect and identify the different faults that may occur in the system over time. VisualBlock-FIR performs these tasks by means of a user-friendly framework. The fault detection process of VisualBlock-FIR is described in Fig. 1.

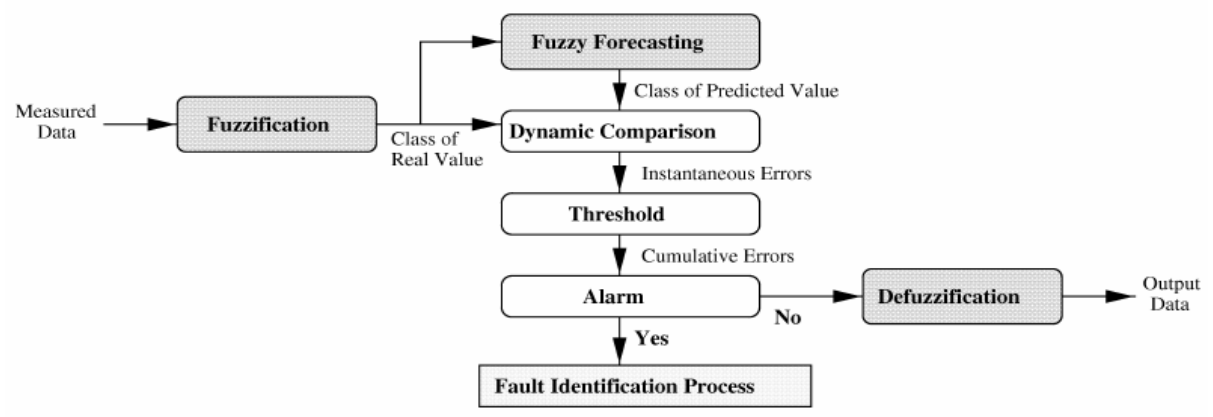

Fig. 1. VisualBlock-FIR Fault Detection Process

In Fig. 1 the grey boxes represent FIR processes, whereas the white boxes constitute the fault detection procedure. The data measured from the system is converted into qualitative triples (class, membership, and side) by means of the FIR fuzzification process. The fuzzy forecasting process predicts the next output value, a qualitative triple, from the qualitative data using the model (mask and pattern rule base) that represents the current behavior of the system. The fuzzy forecasting process computes also the enveloping interval that drives the detection process. The enveloping concept is based on the 5 nearest neighbors that are computed inside the FIR inference engine by means of the k-nearest neighbor rule. A value of 5 has been chosen because from a statistical point of view every state should be observed at least five times [11]. A distance measure is computed between the input pattern, from which the output prediction should be obtained, and all patterns stored in the pattern rule base that match with that input pattern. The 5 patterns with shortest distance are selected as the 5 nearest neighbors. For a deeper insight into the FIR methodology, the reader is referred to [12]. As shown in Fig. 2, the enveloping is composed by an upper bound (maximum value) and a lower bound (minimum value) delimiting the space where the real output signal should be present. It is important to notice that the enveloping 
bounds are obtained directly from the 5 nearest neighbors, and therefore the predicted signal is always in the range set by the two bounds. This is not the case of the real signal. If a real value falls outside the envelope, an instantaneous error occurs, meaning that the model used in the prediction does not correctly represent the system in that specific point. The instantaneous errors occurred inside a predetermined time window are accumulated over time (see Fig. 2). When the cumulative errors within the window are greater than the threshold specified by the modeler, an alarm is issued, and it is then necessary to identify the fault that has occurred. A narrow enveloping interval implies that the 5 neighbors are very close to each other, meaning that the information available of the behavior of the system in that point is very rich and complete. By contrast, a wide enveloping interval means that there is not a lot of information about the system in that point, and therefore, the nearest neighbors are far away from each other. It is important to keep in mind that the FIR methodology is based on the system's behavior rather than on its structure, and therefore, the amount and richness of the data available from the system are crucial in order to assure the identification of an accurate and reliable model representing it.

Fig. 2 presents an example of FIR fault detection using the enveloping concept showing a time window of 15 prediction points. The upper and lower dotted lines represent the upper and lower bounds of the envelope, respectively, whereas the continuous line is the real output signal. In the bottom part of the figure the instantaneous errors are accumulated. As can be seen, the real value falls outside the envelope for the first time in point number 6 when the real value is bigger than the enveloping maximum value, causing an instantaneous error. The same occurs in points number 7 and 11 . The threshold specified in this example was of 3 cumulative errors, and therefore, a fault alarm is triggered in point number 11 when the third instantaneous error arrives. The next step is the identification of the new fault that has occurred.

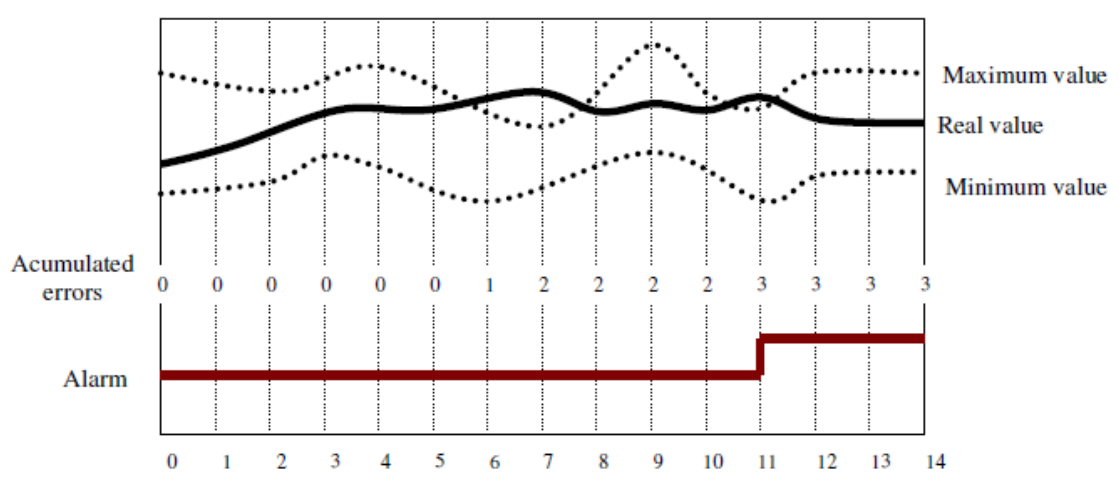

Fig. 2. Example of FIR fault detection using the enveloping method 
The fault identification process is presented in Fig. 3.

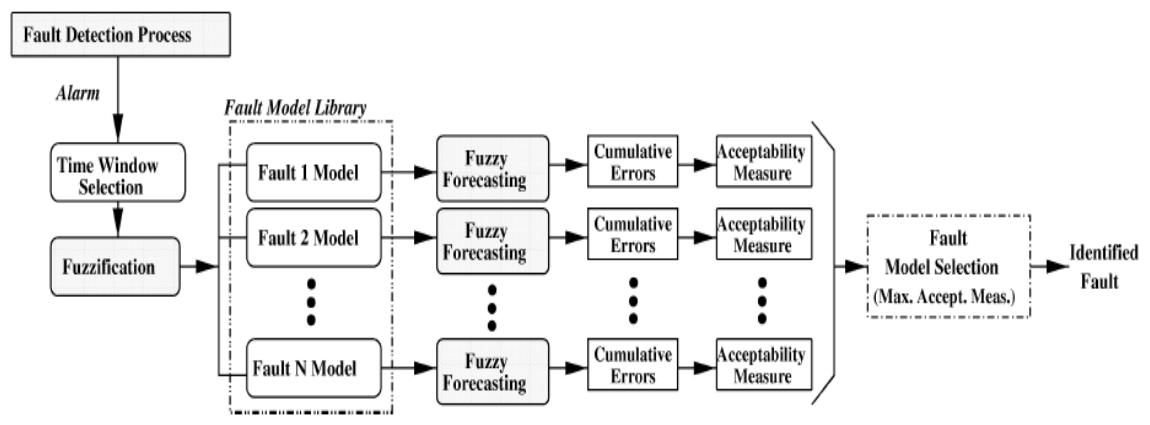

Fig. 3. FIR Fault Identification Process

In Fig. 3, the grey boxes represent FIR processes whereas the white boxes constitute the fault identification procedure. Once the alarm has been triggered because an abnormal behavior has been detected, a time window is selected. The size of the time window defines the number of prediction values that will be used in order to identify the fault that has been produced. Therefore, the time window guides the prediction during the identification process. A small size of the time window is desired because it implies fast model identification. For each fault model stored in the fault model library, a prediction of the size of the time window takes place using the FIR fuzzy forecasting process. The prediction errors produced during each of the forecasting processes are accumulated. Therefore, each fault model stored in the library has associated a cumulative error, $I a_{i}$. This error is used to compute the model acceptability measure. The acceptability measure is a relative index ranking the models in terms of their ability to predict the new behavior of the system. This measure allows us, in a reliable way, to identifyy the fault that has occurred. It also offers guidance when the identification process faces additional problems, e.g. when the produced fault is not a foreseen fault and therefore is not available in the fault model library, or when two different models can be identified that both are able of explaining an observed fault. The acceptability measure of the $\mathrm{i}^{\text {th }}$ model, $Q_{i}$, is described by the following formula:

$$
Q_{i}=C_{i} \cdot C_{r e l_{i}}
$$

where $C_{i}$ is the partial acceptability measure of the $\mathrm{i}^{\text {th }}$ model and $C_{r e l_{i}}$ is a relative confidence that takes the dispersion between the $C_{i}$ values into account. $C_{i}$ is computed by use of the sum of cumulative errors for that particular model and the maximum number of local cumulative errors possible (depends on the size of the time window): 


$$
C_{i}=1.0-I_{a_{i}} / I_{a_{\max }}
$$

and the relative confidence is obtained by the equation:

$$
C_{r e l_{i}}=C_{i} / \sum_{k=1}^{N} C_{k}
$$

The model with the largest acceptability measure is selected as the one that best represents the new behavior of the system, and therefore, the detected fault has been identified.

\section{Case study: Fault Detection and Identification of a Valve Actuator}

This section presents the results of applying VisualBlock-FIR to the DAMADICS actuator benchmark. The industrial actuator consists of a flow servo-valve driven by a smart positioner, as shown in Fig. 4.

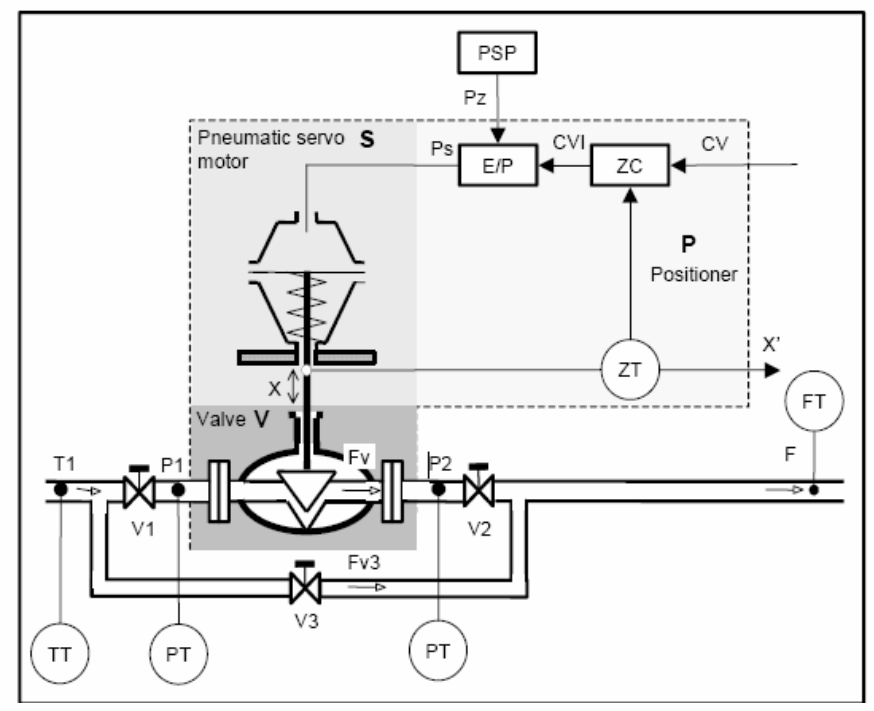

Fig. 4. Schematic representation of the pneumatic servo-motor actuated control valve (Extracted from [8])

The actuator consists of the control valve $(\mathrm{V})$, the pneumatic servomotor $(\mathrm{S})$, and the positioner $(\mathrm{P})$. These three main parts are composed by a set of basic measured physical values, such as flow sensor measurement $(\mathrm{F})$, valve input pressure $(\mathrm{P} 1)$, valve output pressure (P2), liquid temperature (T1), rod displacement (X) and external (flow or level) controller output (CV).

In order to test the robustness of the VisualBlock-FIR approach, two different faults have been simulated following the rules defined in DABLib for benchmark 
purposes. The first fault correspond to fault F10, a servo-motor's diaphragm perforation caused by fatigue of diaphragm material, and the second one correspond to fault $\mathrm{F} 1$, a valve clogging that is a blocking servomotor rod displacement caused by an external mechanical event. Fault F10 corresponds to the pneumatic servomotor part and fault F1 to the control valve part. For both experiments, abrupt small and medium fault scenarios according to the DAMADICS benchmark definition are studied. Therefore, we are in fact dealing with four faults in this research, i.e., F10s, F10m, $\mathrm{F} 1 \mathrm{~s}$, and F1m.

The first step is to obtain the FIR qualitative models that constitute the fault library. In this case, five different models should be identified, before VisualBlock-FIR is ready to perform the fault identification. Therefore, a FIR model for the correct behavior of the plant, as well as models for each type of diaphragm perforation behavior (small and medium) as well as for each type of valve clogging behavior (small and medium), are needed. For each of these scenarios, the system is simulated across 700 seconds of simulation time with a sampling rate of 0.25 seconds, generating 2800 data points. The first 100 seconds (400 data points) are used by FIR to identify the model, and the final 600 seconds (2400 data points) are used to verify the model. All the models are SISO, with $\mathrm{CV}$ as input variable and $\mathrm{X}$ as output variable. The mean square error, $M S E$, in percentage is used to compute the prediction error, as described in equation 4 , where $y(t)$ is the real signal and $\hat{y}(t)$ is the predicted signal.

$$
M S E=\frac{E\left[(y(t)-\hat{y}(t))^{2}\right]}{y_{\mathrm{var}}} .100 \%
$$

An MSE of $4.99 \mathrm{e}^{-2} \%$ is obtained for the non-fault model when it is used to predict the test data set. Errors of $3.22 \mathrm{e}^{-2} \%$ and $2.91 \mathrm{e}^{-2} \%$ are obtained when using the F1s and F1m models, respectively, to predict the test data set. Finally, MSEs of $2.11 \mathrm{e}^{-1} \%$ and $2.34 \mathrm{e}^{-1} \%$ are obtained when using the F10s and F10m models, respectively, to predict the test data set. These prediction errors are very low, meaning that the models obtained represent accurately each of the system's behaviors. The FIR models (masks and behavior matrices) of the four faults as well as the model of the valve working properly are stored in the fault model library to be used during the identification phase of VisualBlock-FIR.

\subsection{Diaphragm Perforation Fault (F10)}

Small and medium diaphragm perforations are introduced at time 200 seconds. Fig. 5 and 6 show the results of the detection process of VisualBlock-FIR for small (F10s) and medium (F10m) fault sizes, respectively. As can be seen from the top plot of both figures, the detection is performed almost instantaneously when the fault occurs. In this case, it is defined that three cumulative errors are needed in order to trigger an alarm. Therefore, for faults F10s and F10m, only 0.75 seconds are needed for VsiualBlock-FIR to determine that the valve is not working properly. At time $200.75 \mathrm{sec}-$ onds an alarm is activated as shown in the top plot of Fig. 5 and 6 (labeled with the word "Fault"). Notice that during the first 200 seconds the predicted signal of the model without fault is completely inside the enveloping interval (top plot of both figures) whereas the predicted signals of F10s and F10m faults are outside that 
interval (second plot of both figures). After second 200 this behavior changes completely, now the predicted signals that are inside the enveloping interval are the ones obtained by means of the fault models. Notice however that the lower curves of the signals are exactly the same for both (fault and non-fault) system behaviors. Therefore, these segments of the signal are always inside the envelope.
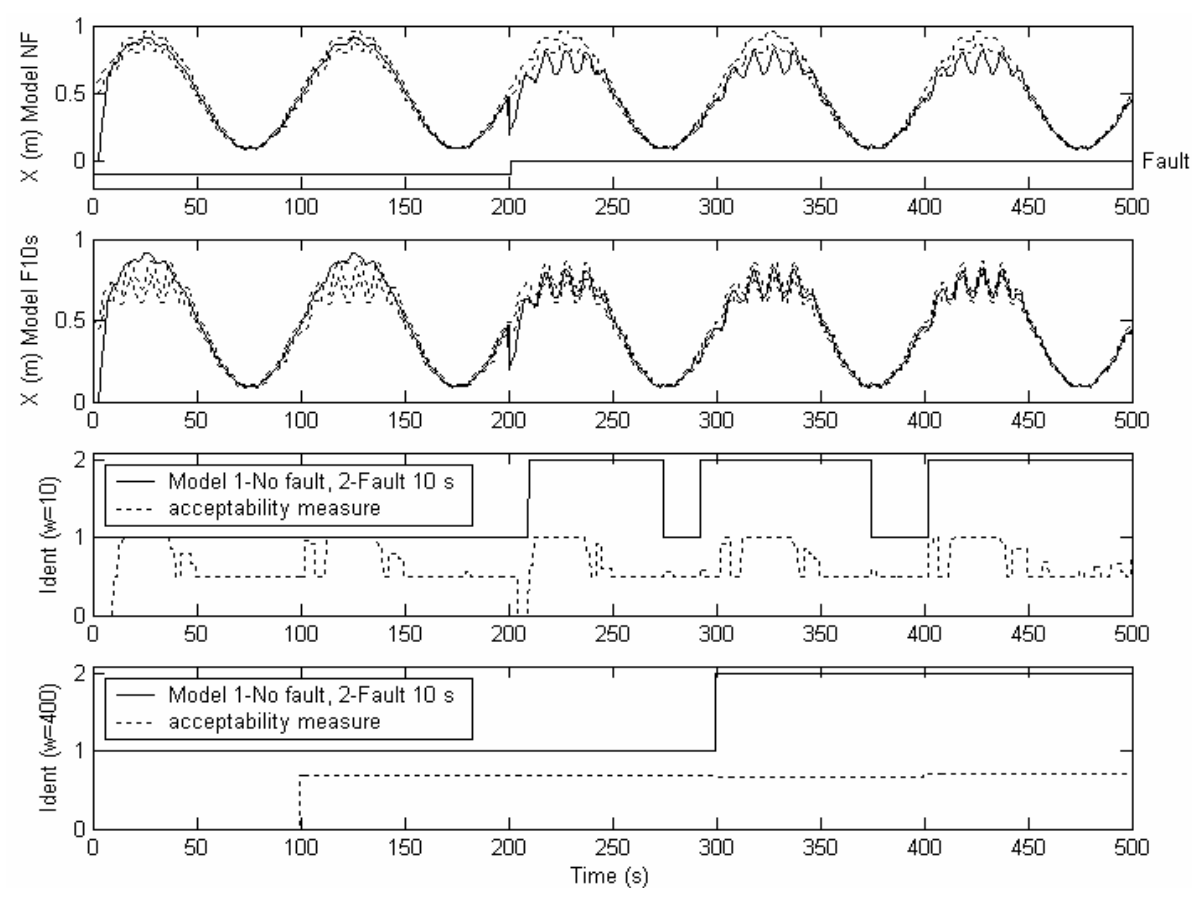

Fig. 5. Fault F10s detection by means of VisualBlock-FIR

Once the alarm has been triggered the identification process starts (see Fig. 3). In this case a time window of 10 data points (corresponding to 2.5 seconds) is used to determine the fault that has occurred. The third plot of both figures show the identification process when a time window of 2.5 seconds is used. After fault detection at time 200.75 seconds, the identification process concludes that the model representing the F10s fault is the one that best corresponds with the actual behavior of the system. Notice however that the identification process fails twice during time periods of 18 seconds and 25 seconds when it decides that the model without fault is the one that represents best the observed behavior of the system. Looking closer at the two periods when the model identification fails, it can be seen that they correspond to the two valleys of the signal, i.e., time segments that both models can predict well because the fault and non-fault behaviors cannot be distinguished during these periods. The lower plot of Fig. 5 shows the identification process when a time window of 400 data points (corresponding to 100 seconds, i.e. a complete signal cycle), is being used. As can be seen, the identification process does an excellent work; during the last 300 seconds it 
identifies the F10s model as the one that best follows the system behavior. Notice that the identification of the fault is produced at time 300 seconds only because the time window is now 100 seconds wide. Notice that during the first 99 seconds the acceptability measure assumes the default value of 0 , until the acceptability measure is computed for the first time after 100 seconds (once the time window period has passed).
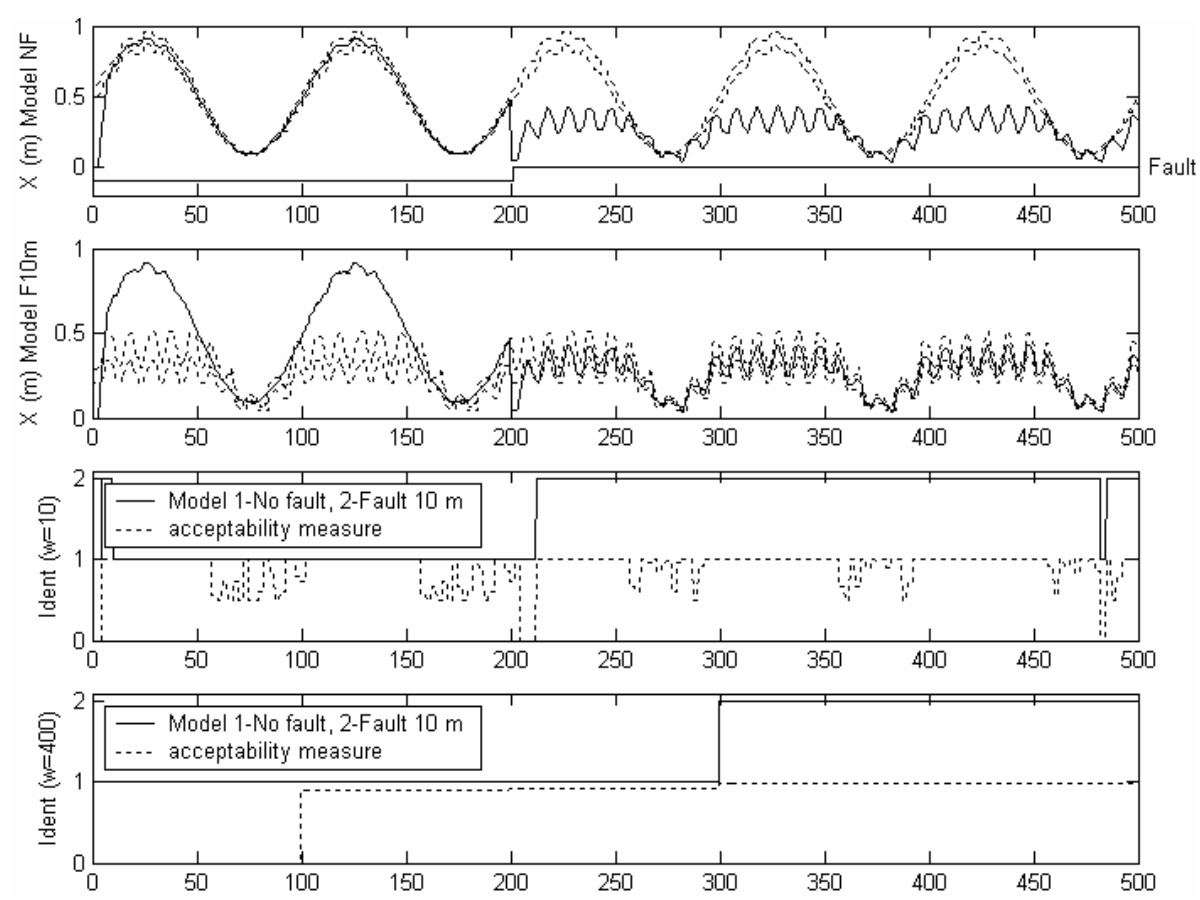

Fig. 6. Fault F10m detection by means of VisualBlock-FIR

The same analysis can be performed for the diaphragm perforation medium fault, F10m, shown in Fig. 6. In this case, the identification process with a time window of 10 data points $(2.5$ seconds $)$ is almost perfect. Only one small mistake of e few seconds duration is encountered. When the time window is increased in such a way that it covers a complete signal cycle, the identification is done properly. Notice that in this case, the signals measured faultless operation of the system and during the presence of a F10m fault are quite different, making the identification task of the VisualBlock-FIR FDS easier.

\subsection{Valve Clogging Fault (F1)}

Small and medium valve clogging faults are introduced at time 200 seconds. Fig. 7 and 8 show the results of the detection process of VisualBlock-FIR for small (F1s) and medium $(\mathrm{F} 1 \mathrm{~m})$ fault sizes, respectively. In both cases, the valleys of the signals are essentially the same for both system behaviors, i.e. with and without a fault, 

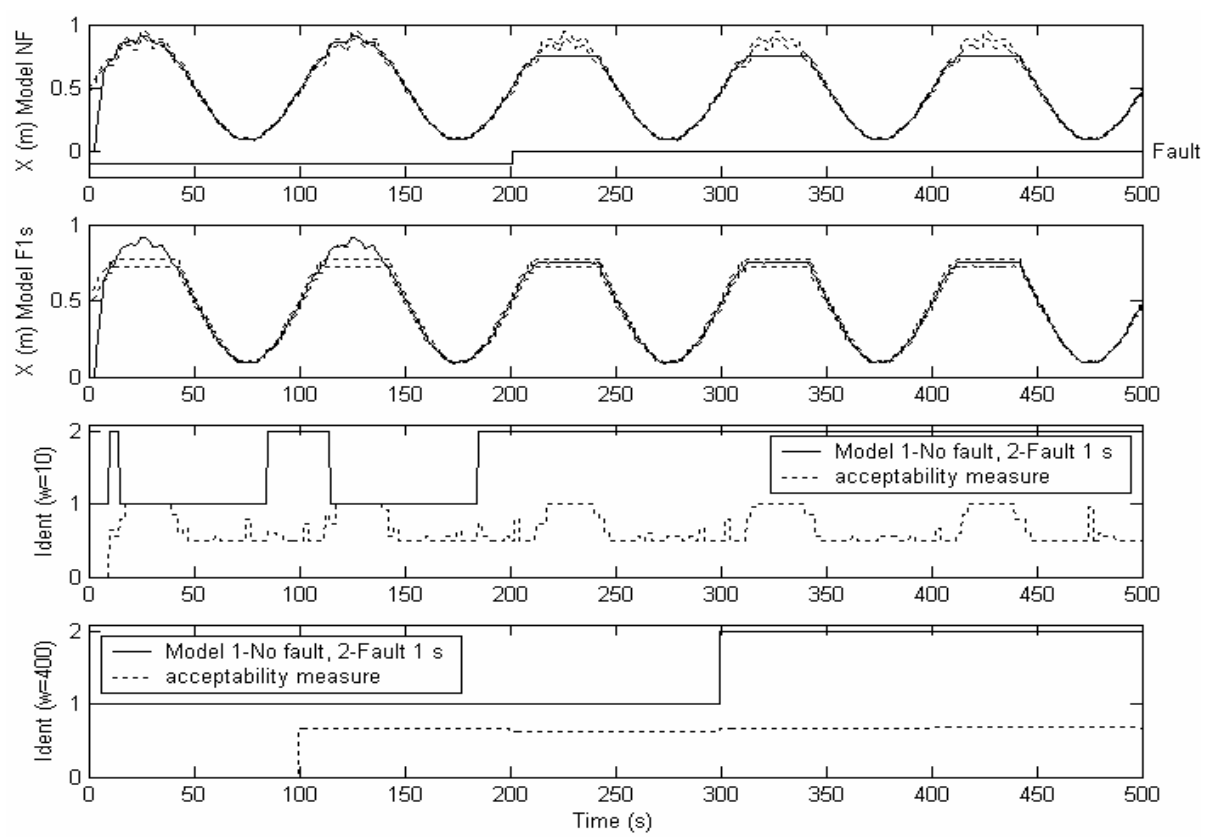

Fig. 7. Fault F1s detection by means of VisualBlock-FIR
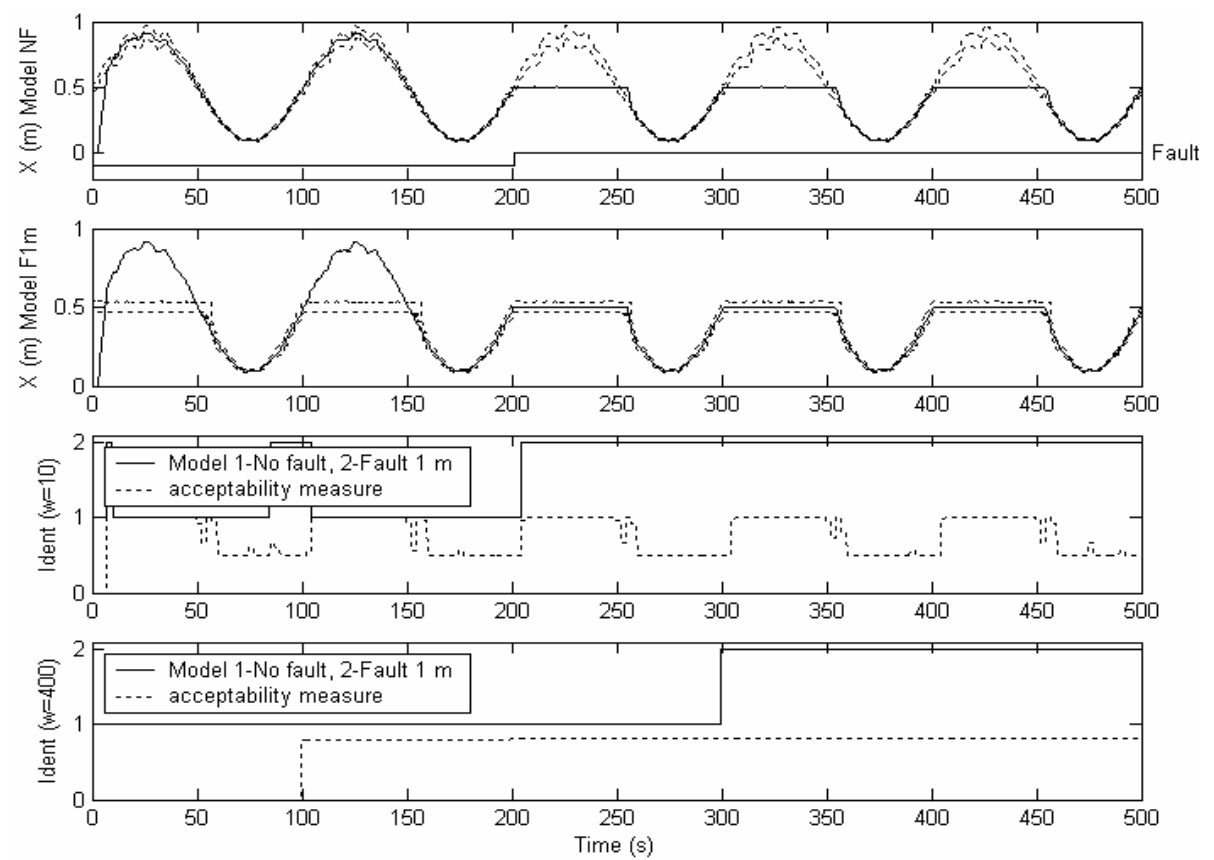

Fig. 8. Fault F1m detection by means of VisualBlock-FIR 
making the identification phase trickier. The two top plots of Fig. 7 and 8 show that the predictions obtained using the model of the system working properly as well as the models of the F1s and F1m faults are very accurate. As has happened in the case of the F10 faults, the detection is performed almost instantaneously when the fault occurs, as shown in the top plots of both figures.

The third plot of Fig. 7 and 8 shows that the fault identification phase of VisualBlock-FIR has three and two errors, respectively, during the first 200 seconds, where the system has no fault. However, the identification is performed very well in the period where the system presents the F1s and F1m faults. In this case, a time window of 10 data points ( 2.5 seconds) is used. When the time window is increased to cover a complete signal cycle, i.e., 400 data points (100 seconds), the identification is done properly as shown in the lower plot of Fig. 7 and 8.

Notice that in this research we have decided to activate the identification process of VisualBlock-FIR from the beginning, in order to better study its performance. However when VisualBlock-FIR is used in a real-world situation, it is recommended to start the identification process only after the detection phase has triggered an alarm that a fault has occurred, i.e. in the experiment at hand at second 200.75. Such a procedure would eliminate the false alarms that occur in the early phase.

From the experiments shown in Fig. 5, 6, 7, and 8, it can be concluded that VisualBlock-FIR is performing a good job detecting in less than 1 second that a fault has occurred. It also obtains good results in the identification phase, due to the fact that only 2.5 seconds are needed to identify the problem after the alarm went off. In a real-world situation, the identified fault would lead to an intervention in order to either correct the fault or, if this cannot be done, to shut down the system.

\section{Conclusions}

The VisualBlock-FIR fault diagnosis system is presented for the first time as a tool for detection and identification of faults for non-linear plants. The detection and identification processes are introduced in this article and are applied to tackle faults of the DAMADICS benchmark problem. Four different faults in the pneumatic servomotor part and the control valve part are detected and identified in an accurate way.

\section{Acknowledgments}

This research was supported by the Consejo Interministerial de Ciencia y Tecnología under project TIN2006-08114.

\section{References}

1. Basseville, M., Nikiforov, I.: Detection of Abrupt Changes: Theory and Applications. Prentice Hall Information and Systems Science Series, USA (1993)

2. Puig, V., Stancu, A., Escobet, T., Nejjari, F., Quevedo, J., Patton, R.J.: Passive robust fault detection using interval observers: Application to the DAMADICS benchmark problem. Control Engineering Practice 14, 621-633 (2006) 
3. Previdi, F., Parisini, T.: Model-free actuator fault detection using a spectral estimation approach: the case of the DAMADICS benchmark problem. Control Engineering Practice 14(6), 635-644 (2006)

4. Crespo, A.: Real-Time Expert Systems. In: Boullart, L., Krijgsman, A., Vingerhoeds, R.A. (eds.) Applications of Artificial Intelligence in Process Control, Pergamon Press, UK (1993)

5. Kandel, A. (ed.): Fuzzy Expert Systems. CRC Press, USA (1992)

6. Witczak, M., Korbicz, J., Mrugalski, M., Patton, R.J.: A GMDH neural network-based approach to robust fault diagnosis: application to the DAMADICS benchmark problem. Control Engineering Practice 14(6), 671-683 (2006)

7. Witczak, M., Patton, R.J., Korbicz, J.: Fault detection with observers and genetic programming: application to the DAMADICS benchmark problem. In: 5th IFAC Symposium on Fault Detection, Supervision and Safety of Technical Processes - SAFEPROCESS 2003, Washington, USA, 2003, pp. 1203-1208 (2003)

8. Calado, J.M.F., Carreira, F.P.N.F., Mendes, M.J.G.C., Sá da Costa, J.M.G., Bartys, M.: Faul detection approach based on fuzzy qualitative reasoning applied to the DAMADICS benchmark problem. In: 5th IFAC Symposium on Fault Detection, Supervision and Safety of Technical Processes - SAFEPROCESS 2003, Washington, USA, pp. 1179-1184 (2003)

9. Rzepiejewski, P., Syfert, M., Jegorov, S.: On-line actuator diagnosis based on neural models and fuzzy reasoning: The DAMADICS benchmark study. In: 5th IFAC Symposium on Fault Detection Supervision and Safety of Technical Processes - SAFEPROCESS 2003, Washington, USA, pp. 1083-1088 (2003)

10. Uppal, F.J., Patton, R.J., Witczak, M.: A hybrid neuro-fuzzy and de-coupling approach applied to the DAMADICS benchmark problem. In: 5th IFAC Symposium on Fault Detection Supervision and Safety of Technical Processes - SAFEPROCESS 2003, Washington, USA, pp. 1059-1064 (2003)

11. Law, A., Kelton, W.: Simulation modeling and analysis, 2nd edn. McGraw-Hill, New York (1991)

12. Nebot, A.: Qualitative modeling and simulation of biomedical systems using FIR, Ph.D., Univ. Polit. Catalunya (1994) 